\title{
A NOTE ON GENERALIZED EQUIVARIANT HOMOTOPY GROUPS
}

\author{
MAREK GOLASIŃSKI \\ Faculty of Mathematics and Computer Science, Nicolaus Copernicus University \\ Chopina 12/18, 8\%-100, Torun, Poland \\ E-mail:marek@mat.uni.torun.pl \\ and \\ Faculty of Mathematics and Computer Science, University of Warmia and Mazury \\ Żotnierska 14, 10-561 Olsztyn, Poland \\ E-mail:marek@matman.uwm.edu.pl

\section{DACIBERG L. GONÇALVES} \\ Dept. de Matemática, IME, Universidade de São Paulo \\ Caixa Postal 66.281, CEP 05311-970, São Paulo, SP, Brasil \\ E-mail: dlgoncal@ime.usp.br \\ PETER N. WONG \\ Department of Mathematics, Bates College \\ Lewiston, ME 04240, U.S.A. \\ E-mail:pwong@bates.edu
}

\begin{abstract}
In this paper, we generalize the equivariant homotopy groups or equivalently the Rhodes groups. We establish a short exact sequence relating the generalized Rhodes groups and the generalized Fox homotopy groups and we introduce $\Gamma$-Rhodes groups, where $\Gamma$ admits a certain co-grouplike structure. Evaluation subgroups of $\Gamma$-Rhodes groups are discussed.
\end{abstract}

1. Introduction. In 1966, F. Rhodes [8] introduced the fundamental group of a transformation group $(X, G)$ for a topological space on which a group $G$ acts. This group, denoted by $\sigma_{1}\left(X, x_{0}, G\right)$, is the equivariant analog of the classical fundamental group $\pi_{1}\left(X, x_{0}\right)$. Rhodes showed that $\sigma_{1}\left(X, x_{0}, G\right)$ is a group extension of $\pi_{1}\left(X, x_{0}\right)$ with quo-

2000 Mathematics Subject Classification: Primary 55Q05, 55Q15, 55Q91; Secondary 55M20.

Key words and phrases: equivariant maps, Fox torus homotopy groups, generalized Rhodes' groups, homotopy groups of a transformation group.

The paper is in final form and no version of it will be published elsewhere. 
tient $G$. Thus, $\sigma_{1}\left(X, x_{0}, G\right)$ incorporates the $G$-action as well as the action of $\pi_{1}\left(X, x_{0}\right)$ on the universal cover $\tilde{X}$ of the space $X$. This group has been used in [10] to study the Nielsen fixed point theory for equivariant maps. In 1969, F. Rhodes [9] extended $\sigma_{1}\left(X, x_{0}, G\right)$ to $\sigma_{n}\left(X, x_{0}, G\right)$, which is the equivariant higher homotopy group of $(X, G)$. Like $\sigma_{1}\left(X, x_{0}, G\right), \sigma_{n}\left(X, x_{0}, G\right)$ is an extension of the Fox torus homotopy group $\tau_{n}\left(X, x_{0}\right)$ but not of the classical homotopy group $\pi_{n}\left(X, x_{0}\right)$ by $G$. The Fox torus homotopy groups were first introduced by R. Fox [2] in 1948 in order to give a geometric interpretation of the classical Whitehead product. Recently, a modern treatment of $\tau_{n}\left(X, x_{0}\right)$ and of $\sigma_{n}\left(X, x_{0}, G\right)$ has been given in [4] and in [5], respectively. In [5], we further investigated the relationships between the Gottlieb groups of a space and of its orbit space, analogous to the similar study in [3]. Further properties of the Fox torus homotopy groups, their generalizations, and Jacobi identities were studied in [6]. It is therefore natural to generalize $\sigma_{n}\left(X, x_{0}, G\right)$ to more general constructions with respect to general spaces and to co-grouplike spaces $\Gamma$ other than the 1 -sphere $\mathbb{S}^{1}$.

The main objective of this paper is to generalize $\sigma_{n}\left(X, x_{0}, G\right)$ of a $G$-space $X$ with respect to a space $W$ and also with respect to a pair $(W, \Gamma)$, where $W$ is a space and $\Gamma$ satisfies a suitable notion of the classical co-grouplike space. We prove in section 1 that the Rhodes exact sequence of $[9]$ can be generalized to $\sigma_{W}\left(X, x_{0}, G\right):=\{[f ; g] \mid$ $\left.f:\left(\widehat{\Sigma} W, v_{1}, v_{2}\right) \rightarrow\left(X, x_{0}, g x_{0}\right)\right\}$, the $W$-Rhodes group, with the generalized Fox torus homotopy group $\tau_{W}\left(X, x_{0}\right)$ as the kernel. In section 2 , we further extend the construction of Rhodes groups to $\sigma_{W}^{\Gamma}\left(X, x_{0}, G\right):=\left\{[f ; g] \mid f:\left(\Gamma(W), \bar{\gamma}_{1}, \bar{\gamma}_{2}\right) \rightarrow\left(X, x_{0}, g x_{0}\right)\right\}$, the $W$ $\Gamma$-Rhodes groups, where $\Gamma$ admits a co-grouplike structure with two basepoints. Under such assumptions, we obtain a $W$ - $\Gamma$-generalization of the Rhodes exact sequence [9]. In the last section, we generalize the notion of the Gottlieb (evaluation) subgroup to that of a $W$ - $\Gamma$-Rhodes group and we establish a short exact sequence generalizing [5, Theorem 2.2]. Throughout, $G$ denotes a group acting on a compactly generated Hausdorff pathconnected space $X$ with a basepoint $x_{0}$. The associated pair $(X, G)$ is called in the literature a transformation group.

2. Generalized Rhodes groups. For $n \geq 1$, F. Rhodes [9] defined higher homotopy groups $\sigma_{n}\left(X, x_{0}, G\right)$ of a pair $(X, G)$ which is an extension of $\tau_{n}\left(X, x_{0}\right)$ by $G$ so that

$$
1 \rightarrow \tau_{n}\left(X, x_{0}\right) \rightarrow \sigma_{n}\left(X, x_{0}, G\right) \rightarrow G \rightarrow 1
$$

is exact. Here, $\tau_{n}\left(X, x_{0}\right)$ denotes the $n$-th torus homotopy group of $X$ introduced by R. Fox [2]. The group $\tau_{n}=\tau_{n}\left(X, x_{0}\right)$ is defined to be the fundamental group of the function space $X^{\mathbb{T}^{n-1}}$ and is uniquely determined by the groups $\tau_{1}, \tau_{2}, \ldots, \tau_{n-1}$ and the Whitehead products, where $\mathbb{T}^{n-1}$ is the $(n-1)$-dimensional torus. The group $\tau_{n}$ is nonabelian in general.

Now we recall the construction of $\sigma_{n}\left(X, x_{0}, G\right)$ presented in [9]. Suppose that $X$ is a $G$-space with a basepoint $x_{0} \in X$ and let $C_{n}=I \times \mathbb{T}^{n-1}$. We say that a map $f: C_{n} \rightarrow X$ is of order $g \in G$ provided $f\left(0, t_{2}, \ldots, t_{n}\right)=x_{0}$ and $f\left(1, t_{2}, \ldots, t_{n}\right)=g\left(x_{0}\right)$ for $\left(t_{2}, \ldots, t_{n}\right) \in \mathbb{T}^{n-1}$. Two maps $f_{0}, f_{1}: C^{n} \rightarrow X$ of order $g$ are said to be homotopic if there exists a continuous map $F: C^{n} \times I \rightarrow X$ such that: 
- $F\left(t, t_{2}, \ldots, t_{n}, 0\right)=f_{0}\left(t, t_{2}, \ldots, t_{n}\right)$;

- $F\left(t, t_{2}, \ldots, t_{n}, 1\right)=f_{1}\left(t, t_{2}, \ldots, t_{n}\right)$;

- $F\left(0, t_{2}, \ldots, t_{n}, s\right)=x_{0}$

- $F\left(1, t_{2}, \ldots, t_{n}, s\right)=g x_{0}$ for all $\left(t_{2}, \ldots, t_{n}\right) \in \mathbb{T}^{n-1}$ and $s, t \in \mathrm{I}$.

Denote by $[f ; g]$ the homotopy class of a map $f: C_{n} \rightarrow X$ of order $g$ and by $\sigma_{n}\left(X, x_{0}, G\right)$ the set of all such homotopy classes. We define an operation $*$ on the set $\sigma_{n}\left(X, x_{0}, G\right)$ by

$$
\left[f^{\prime} ; g^{\prime}\right] *[f ; g]:=\left[f^{\prime}+g^{\prime} f ; g^{\prime} g\right] .
$$

This operation makes $\sigma_{n}\left(X, x_{0}, G\right)$ a group.

We have generalized the Fox torus homotopy groups in [4]. In this section, we give a similar generalization of Rhodes groups. In a special case, we obtain an extension group of the Abe group considered in [1].

Let $X$ be a path-connected space with a basepoint $x_{0}$. For any space $W$, we let

$$
\sigma_{W}\left(X, x_{0}, G\right):=\left\{[f ; g] \mid f:\left(\widehat{\Sigma} W, v_{1}, v_{2}\right) \rightarrow\left(X, x_{0}, g x_{0}\right)\right\}
$$

where $[f ; g]$ denotes the homotopy class of the map $f$ of order $g \in G, v_{1}$ and $v_{2}$ are the vertices of the cones $C^{+} W$ and $C^{-} W$, respectively and $\widehat{\Sigma} W=C^{+} W \cup C^{-} W$. Under the operation $\left[f_{1} ; g_{1}\right] *\left[f_{2} ; g_{2}\right]:=\left[f_{1}+g_{1} f_{2} ; g_{1} g_{2}\right], \sigma_{W}$ is a group called a $W$-Rhodes group .

Write $C(W, X)$ for the mapping space of all continuous maps from $W$ to $X$ with the compact-open topology. We point out that $\sigma_{W}\left(X, x_{0}, G\right)=\sigma_{1}\left(C(W, X), \overline{x_{0}}, G\right)$ provided $W$ is a locally-compact space, where $(g f)(x)=g f(x)$ for $f \in C(W, X), g \in G$ and $\overline{x_{0}}$ denotes the constant map from $C(W, X)$ determined by the point $x_{0} \in X$.

The canonical projection $\sigma_{W}\left(X, x_{0}, G\right) \rightarrow G$ given by $[f ; g] \mapsto g$ has the kernel $\{[f ; 1] \mid$ $\left.f:\left(\widehat{\Sigma} W, v_{1}, v_{2}\right) \rightarrow\left(X, x_{0}, x_{0}\right)\right\}$. It is easy to see that this kernel is isomorphic to the generalized Fox torus group $[\Sigma(W \sqcup *), X]=\tau_{W}\left(X, x_{0}\right)$ defined in [4]. Therefore, we get the following result.

THEOREM 1. The sequence

$$
1 \rightarrow \tau_{W}\left(X, x_{0}\right) \rightarrow \sigma_{W}\left(X, x_{0}, G\right) \rightarrow G \rightarrow 1
$$

is exact.

REMARK 1. When $W=\mathbb{T}^{n-1}$, the $(n-1)$-dimensional torus, $\sigma_{W}$ coincides with the $n$-th Rhodes group $\sigma_{n}$ and (2) reduces to (1). When $W=\mathbb{S}^{n-1}$, the $(n-1)$-sphere, $\tau_{W}$ becomes $\kappa_{n}$, the $n$-th Abe group (see [2] or [4]). Thus, by Theorem 1, we have the exact sequence

$$
1 \rightarrow \pi_{n}\left(X, x_{0}\right) \rtimes \pi_{1}\left(X, x_{0}\right) \cong \kappa_{n}\left(X, x_{0}\right) \rightarrow \sigma_{\mathbb{S}^{n-1}}\left(X, x_{0}, G\right) \rightarrow G \rightarrow 1 .
$$

One can also generalize the split exact sequence for Rhodes groups from [9] as follows. THEOREM 2. Let $W$ be a space with a basepoint $w_{0}$. Then, for any space $V$, the sequence

$$
1 \rightarrow[(V \times W) / V, \Omega X] \rightarrow \sigma_{V \times W}\left(X, x_{0}, G\right) \stackrel{\leftrightarrow-}{\rightarrow} \sigma_{V}\left(X, x_{0}, G\right) \rightarrow 1
$$

is split exact.

Proof. By [4, Theorem 3.1], we have the split exact sequence

$$
1 \rightarrow[(V \times W) / V, \Omega X] \rightarrow \tau_{V \times W}(X) \stackrel{\leftrightarrow--}{\rightarrow} \tau_{V}(X) \rightarrow 1 .
$$


Given $[F ; g] \in \sigma_{V \times W}\left(X, x_{0}, G\right)$, where $F: \widehat{\Sigma}(V \times W) \rightarrow X$, let $f: \widehat{\Sigma} V \rightarrow X$ be the composite map of $\widehat{\Sigma} V \approx \widehat{\Sigma}\left(V \times\left\{w_{0}\right\}\right) \rightarrow \widehat{\Sigma}(V \times W)$ with $F$. This map gives rise to a homomorphism $\sigma_{V \times W}\left(X, x_{0}, G\right) \rightarrow \sigma_{V}\left(X, x_{0}, G\right)$. Likewise, using the projection $V \times W \rightarrow V$, one obtains a section $\sigma_{V}\left(X, x_{0}, G\right) \rightarrow \sigma_{V \times W}\left(X, x_{0}, G\right)$. We have the commutative diagram

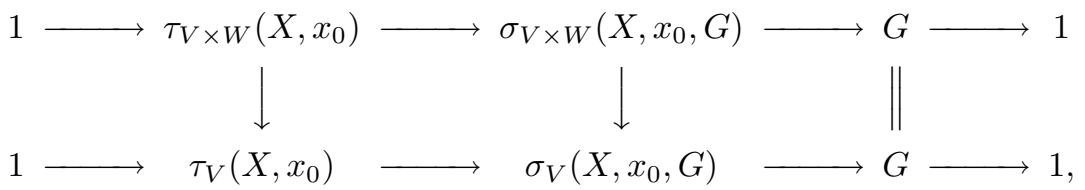

where the first two vertical homomorphisms have sections. Combining with (5), the assertion follows.

As an immediate corollary of Theorem 2, we have the following:

Corollary 3. The sequence

$$
1 \rightarrow[W, \Omega X] \rightarrow \sigma_{W}\left(X, x_{0}, G\right) \stackrel{\leftrightarrow--}{\rightarrow} \sigma_{1}\left(X, x_{0}, G\right) \rightarrow 1
$$

is split exact.

Proof. The result follows from Theorem 2 by letting $V$ be a point.

REMARK 2. For any space $W$, Corollary 3 asserts that $\sigma_{1}\left(X, x_{0}, G\right)$ acts on $[\Sigma W, X]=$ $[W, \Omega X]$ according to the splitting. Furthermore, when $W=\mathbb{S}^{n-1}$, this corollary gives an alternate description of the action of $\sigma_{1}$ on $\pi_{n}(X)$ as described in [5, Remark 1.4]. In this case, $\sigma_{W}\left(X, x_{0}, G\right)=\sigma_{\mathbb{S}^{n-1}}\left(X, x_{0}, G\right)$ is the extension group of the $n$-th Abe group $\kappa_{n}\left(X, x_{0}\right)$ [1] as in (3). Thus, one can either embed $\sigma_{1}$ in $\sigma_{n}$ as in [5, Remark 1.4] or in $\sigma_{\mathbb{S}^{n-1}}\left(X, x_{0}, G\right)$.

Unlike the reduced suspension $\Sigma$ which has the loop functor $\Omega$ as its right adjoint, the un-reduced suspension $\widehat{\Sigma}$ does not admit a right adjoint. Nevertheless, one can describe the adjoint property for the $W$-Rhodes groups as follows. Recall that a typical element in $\sigma_{W}\left(X, x_{0}, G\right)$ is a homotopy class $[f ; g]$ where $f:\left(\widehat{\Sigma} W, v_{1}, v_{2}\right) \rightarrow\left(X, x_{0}, g x_{0}\right)$. Thus, $\sigma_{W}$ is a subset of $[\widehat{\Sigma} W, X]_{0} \times G$, where $[\widehat{\Sigma} W, X]_{0}$ denotes the homotopy classes of maps $f: \widehat{\Sigma} W \rightarrow X$ such that $f\left(v_{1}\right)=x_{0}$ and $f\left(v_{2}\right)$ is independent of the homotopy class of $f$. Then, $\sigma_{W}$ is also a subset of $\left[W, \mathcal{P}_{x_{0}}\right]^{*} \times G$, where $\left[W, \mathcal{P}_{x_{0}}\right]^{*}$ denotes the set of homotopy classes of unpointed maps from $W$ to the space $\mathcal{P}_{x_{0}}$ of paths originating from $x_{0}$. In the special case when $G=\{1\}, \sigma_{W}=[\Sigma(W \cup *), X]=\sigma_{W}^{*}=[W, \Omega Y]^{*}=[W \cup *, \Omega X]$.

3. Generalized $W$ - $\Gamma$-Rhodes groups. In the definition of the generalized Rhodes group $\sigma_{W}\left(X, x_{0}, G\right)$, the two cone points from the un-reduced suspension $\widehat{\Sigma} W=C^{+} W \cup$ $C^{-} W$ play an important role. Therefore in replacing $\mathbb{S}^{1}$ with arbitrary co-grouplike space, we require that the space has two distinct basepoints.

Let $\Gamma$ be a space and $\gamma_{1}, \gamma_{2} \in \Gamma$ satisfying the following conditions:

(I) there exists a map $\nu:\left(\Gamma, \gamma_{1}, \gamma_{2}\right) \rightarrow\left(\Gamma \times\left\{\gamma_{1}\right\} \cup\left\{\gamma_{2}\right\} \times \Gamma,\left(\gamma_{1}, \gamma_{1}\right),\left(\gamma_{2}, \gamma_{2}\right)\right)$ such that $\operatorname{proj}_{i} \circ \nu \simeq \mathrm{id}$ as maps of triples for each $i=1,2$, where $\operatorname{proj}_{1}$, $\operatorname{proj}_{2}:\left(\Gamma \times\left\{\gamma_{1}\right\} \cup\right.$ $\left.\left\{\gamma_{2}\right\} \times \Gamma,\left(\gamma_{1}, \gamma_{1}\right),\left(\gamma_{2}, \gamma_{2}\right)\right) \rightarrow\left(\Gamma, \gamma_{1}, \gamma_{2}\right)$ are the canonical projections; 
(II) there exists a map $\eta: \Gamma \rightarrow \Gamma$ such that:

(a) $\eta\left(\gamma_{1}\right)=\gamma_{2}, \eta\left(\gamma_{2}\right)=\gamma_{1}$;

(b) $\nabla \circ(\overline{\mathrm{id}} \vee \bar{\eta}) \circ \nu$ is homotopic to the constant map at $\gamma_{1}$, where

$\overline{\mathrm{id}} \vee \bar{\eta}: \Gamma \times\left\{\gamma_{1}\right\} \cup\left\{\gamma_{2}\right\} \times \Gamma,\left(\gamma_{1}, \gamma_{1}\right),\left(\gamma_{2}, \gamma_{2}\right) \rightarrow \Gamma \times\left\{\gamma_{1}\right\} \cup\left\{\gamma_{2}\right\} \times \Gamma,\left(\gamma_{1}, \gamma_{2}\right),\left(\gamma_{2}, \gamma_{1}\right)$ with $\overline{\operatorname{id}}\left(\gamma, \gamma_{1}\right)=\left(\gamma, \gamma_{2}\right), \bar{\eta}\left(\gamma_{2}, \gamma\right)=\left(\gamma_{2}, \eta(\gamma)\right)$ for $\gamma \in \Gamma$ and $\nabla:\left(\Gamma \times\left\{\gamma_{2}\right\} \cup\left\{\gamma_{2}\right\} \times\right.$ $\left.\Gamma,\left(\gamma_{1}, \gamma_{2}\right),\left(\gamma_{2}, \gamma_{1}\right)\right) \rightarrow\left(\Gamma, \gamma_{1}, \gamma_{2}\right)$ is the folding map;

(c) similarly, $\nabla \circ(\tilde{i d} \vee \tilde{\eta}) \circ \nu$ is homotopic to the constant map at $\gamma_{2}$, where id $\vee \tilde{\eta}: \Gamma \times\left\{\gamma_{1}\right\} \cup\left\{\gamma_{2}\right\} \times \Gamma,\left(\gamma_{1}, \gamma_{1}\right),\left(\gamma_{2}, \gamma_{2}\right) \rightarrow \Gamma \times\left\{\gamma_{1}\right\} \cup\left\{\gamma_{2}\right\} \times \Gamma,\left(\gamma_{2}, \gamma_{1}\right),\left(\gamma_{1}, \gamma_{2}\right)$ with $\tilde{\mathrm{id}}\left(\gamma_{2}, \gamma\right)=\left(\gamma_{1}, \gamma\right), \tilde{\eta}\left(\gamma, \gamma_{1}\right)=\left(\eta(\gamma),\left(\gamma_{1}\right)\right)$ for $\gamma \in \Gamma$;

(III) Moreover, we have co-associativity so that the diagram

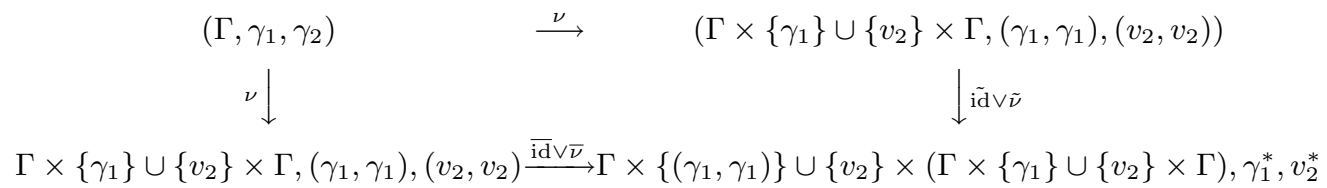

is commutative up to homotopy, where $\gamma_{1}^{*}=\left(\gamma_{1},\left(\gamma_{1}, \gamma_{1}\right)\right)$, $\gamma_{2}^{*}=\left(\gamma_{2},\left(\gamma_{2}, \gamma_{2}\right)\right)$, and $\left.\overline{\mathrm{id}}\left(\gamma, \gamma_{1}\right)=\left(\gamma,\left(\gamma_{1}, \gamma_{1}\right)\right), \bar{\nu}\left(\gamma_{2}, \gamma\right)=\left(\gamma_{2}, \nu(\gamma)\right), \tilde{\operatorname{id}}\left(\gamma_{2}, \gamma\right)=\left(\left(\gamma_{2}, \gamma_{2}\right), \gamma\right)\right), \tilde{\nu}\left(\gamma, \gamma_{1}\right)=$ $\left(\nu(\gamma), \gamma_{1}\right)$ for $\gamma \in \Gamma$.

Now, we generalize the notion of a co-grouplike space presented e.g. in [7]. A cogrouplike space with two basepoints $\Gamma=\left(\Gamma, \gamma_{1}, \gamma_{2} ; \nu, \eta\right)$ consists of a topological space $\Gamma$ together with basepoints $\gamma_{1}, \gamma_{2}$ and maps $\nu, \eta$ satisfying conditions (I)-(III). For any space $W$, the smash product is given by

$$
\Gamma(W):=W \times \Gamma /\left\{\left(w, \gamma_{1}\right) \sim\left(w^{\prime}, \gamma_{1}\right),\left(w, \gamma_{2}\right) \sim\left(w^{\prime}, \gamma_{2}\right)\right\}
$$

for any $w, w^{\prime} \in W$.

For instance, if $\Gamma=([0,1], 0,1 ; \nu, \eta)$ with $\nu(t)=\left\{\begin{array}{ll}(2 t, 0) & \text { if } 0 \leq t \leq \frac{1}{2}, \\ (1,2 t-1) & \text { if } \frac{1}{2} \leq t \leq 1\end{array}\right.$ and $\eta(t)=1-t$ for $t \in[0,1]$ then $\Gamma(W)=\widehat{\Sigma} W$, the un-reduced suspension of $W$.

REMARK 3. Note that if $\gamma_{1}=\gamma_{2}$, we obtain the usual co-grouplike structure and $\Gamma_{0}:=$ $\Gamma / \sim$ given by identifying the basepoints $\gamma_{1}$ and $\gamma_{2}$ is a co-grouplike space as well.

Next, we define the $W$ - $\Gamma$-Rhodes groups.

Let $\Gamma$ be a co-grouplike space with two basepoints, $(X, G)$ a $G$-space and $W$ a space. The $W$ - $\Gamma$-Rhodes group of $X$ with respect to $W$ is defined to be

$$
\sigma_{W}^{\Gamma}\left(X, x_{0}, G\right)=\left\{[f ; g] \mid f:\left(\Gamma(W), \bar{\gamma}_{1}, \bar{\gamma}_{2}\right) \rightarrow\left(X, x_{0}, g x_{0}\right)\right\} .
$$

Write $\tau_{W}^{\Gamma_{0}}\left(X, x_{0}\right)$ for the $\Gamma_{0}-W$-Fox group considered in [6].

We can easily show:

Proposition 4. Let $\pi: \sigma_{W}^{\Gamma}\left(X, x_{0}, G\right) \rightarrow G$ be the projection sending $[f ; g] \mapsto g$. By identifying the two basepoints of $\Gamma(W)$, the quotient space $\Gamma(W) / \sim$ is canonically homeomorphic to $\Gamma_{0} \wedge(W \cup\{*\})$. Furthermore,

$$
\operatorname{Ker} \pi \cong[\Gamma(W) / \sim, X]=\tau_{W}^{\Gamma_{0}}\left(X, x_{0}\right) .
$$


Then we obtain a general $\Gamma$-Rhodes exact sequence, generalizing (2).

THEOREM 5. The sequence

$$
1 \rightarrow \tau_{W}^{\Gamma_{0}}\left(X, x_{0}\right) \rightarrow \sigma_{W}^{\Gamma}\left(X, x_{0}, G\right) \stackrel{\pi}{\rightarrow} G \rightarrow 1
$$

is exact.

We now derive the following generalized split exact sequence for the $W$ - $\Gamma$-Rhodes groups.

Corollary 6. Let $W$ be a space with a basepoint $w_{0}$ and $\Gamma$ be a co-grouplike space with two basepoints. The sequence

$$
1 \rightarrow\left[\Gamma_{0} \wedge((V \times W) / V), \Omega X\right] \rightarrow \sigma_{V \times W}^{\Gamma}\left(X, x_{0}, G\right) \stackrel{\leftrightarrow--}{\rightarrow} \sigma_{V}^{\Gamma}\left(X, x_{0}, G\right) \rightarrow 1
$$

is split exact.

Proof. From Theorem 5, we have the short exact sequences

$$
1 \rightarrow \tau_{V \times W}^{\Gamma_{0}}\left(X, x_{0}\right) \rightarrow \sigma_{V \times W}^{\Gamma}\left(X, x_{0}, G\right) \stackrel{\pi}{\rightarrow} G \rightarrow 1
$$

and

$$
1 \rightarrow \tau_{V}^{\Gamma_{0}}\left(X, x_{0}\right) \rightarrow \sigma_{V}^{\Gamma}\left(X, x_{0}, G\right) \stackrel{\pi}{\rightarrow} G \rightarrow 1 .
$$

Moreover, the following split exact sequence was shown in [6, Theorem 4.1]:

$$
1 \rightarrow\left[\Gamma_{0} \wedge((V \times W) / V), \Omega X\right] \rightarrow \tau_{V \times W}^{\Gamma_{0}}\left(X, x_{0}, G\right) \stackrel{\leftrightarrow--}{\rightarrow} \tau_{V}^{\Gamma_{0}}\left(X, x_{0}, G\right) \rightarrow 1 .
$$

A straightforward diagram chasing argument involving these short exact sequences yields the desired split exact sequence.

4. Evaluation subgroups of $W$ - $\Gamma$-Rhodes groups. We end this note by extending a result concerning the evaluation subgroups of the Rhodes groups and the Fox torus homotopy groups obtained in [5, Theorem 2.2].

Given a $G$-space $X$, the function space $X^{X}$ is also a $G$-space where the action is pointwise, that is, $(g f)(x)=g f(x)$ for $f \in X^{X}, g \in G$ and $x \in X$. Let $\Gamma$ be a co-grouplike space with two basepoints and $W$ be a space.

The evaluation subgroup of the $W$ - $\Gamma$-Rhodes group of $X$ is defined by

$$
\mathcal{G} \sigma_{W}^{\Gamma}\left(X, x_{0}, G\right):=\operatorname{Im}\left(e v_{*}: \sigma_{W}^{\Gamma}\left(X^{X}, \mathrm{id}_{X}, G\right) \rightarrow \sigma_{W}^{\Gamma}\left(X, x_{0}, G\right)\right) .
$$

Similarly, the evaluation subgroup of $\tau_{W}^{\Gamma_{0}}\left(X, x_{0}\right)$ is defined by

$$
\mathcal{G} \tau_{W}^{\Gamma_{0}}\left(X, x_{0}\right):=\operatorname{Im}\left(e v_{*}: \tau_{W}^{\Gamma_{0}}\left(X^{X}, \operatorname{id}_{X}\right) \rightarrow \tau_{W}^{\Gamma_{0}}\left(X, x_{0}\right)\right)
$$

It is straightforward to see that the proof of [5, Theorem 2.2] is also valid in the setting of $W$ - $\Gamma$-Rhodes groups. Therefore, we have the following generalization.

THEOREM 7. Let $G_{0}$ be the subgroup of $G$ consisting of elements $g$ considered as homeomorphisms of $X$ which are freely homotopic to the identity map $\mathrm{id}_{X}$. Then the sequence

$$
1 \rightarrow \mathcal{G} \tau_{W}^{\Gamma_{0}}\left(X, x_{0}\right) \rightarrow \mathcal{G} \sigma_{W}^{\Gamma}\left(X, x_{0}, G\right) \rightarrow G_{0} \rightarrow 1
$$

is exact. 
Acknowledgements. This work was conducted during the second and third authors' visit to the Faculty of Mathematics and Computer Science, Nicolaus Copernicus University, July 27 - 31, 2007. These authors would like to thank the Faculty of Mathematics and Computer Science for its hospitality and support.

\section{References}

[1] M. Abe, Über die stetigen Abbildungen der $n$-Sphäre in einen metrischen Raum, Japanese J. Math. 16 (1940), 169-176.

[2] R. Fox, Homotopy groups and torus homotopy groups, Ann. of Math. 49 (1948), 471-510.

[3] M. Golasiński and D. Gonçalves, Postnikov towers and Gottlieb groups of orbit spaces, Pacific J. Math. 197 (2001), 291-300.

[4] M. Golasiński, D. Gonçalves and P. Wong, Generalizations of Fox homotopy groups, Whitehead products, and Gottlieb groups, Ukrain. Mat. Zh. 57 (2005), 320-328 (in Russian); English transl.: Ukrainian Math. J. 57 (2005), 382-393.

[5] M. Golasiński, D. Gonçalves and P. Wong, Equivariant evaluation subgroups and Rhodes groups, Cah. Topol. Géom. Différ. Catég. 48 (2007), 55-69.

[6] M. Golasiński, D. Gonçalves and P. Wong, On Fox spaces and Jacobi identities, Math. J. Okayama Univ. 50 (2008), 161-176.

[7] N. Oda and T. Shimizu, A $\Gamma$-Whitehead product for track groups and its dual, Quaest. Math. 23 (2000), 113-128.

[8] F. Rhodes, On the fundamental group of a transformation group, Proc. London Math. Soc. 16 (1966), 635-650.

[9] F. Rhodes, Homotopy groups of transformation groups, Canad. J. Math. 21 (1969), 11231136.

[10] P. Wong, Equivariant Nielsen numbers, Pacific J. Math. 159 (1993), 153-175. 
